\title{
Service Logic Mobility over Intelligent Broadband Networks
}

\author{
Ch. Z. Patrikakis, S. E. Polykalas, I. S. Venieris
}

Electrical \& Computer Engineering Department

National Technical University of Athens

9 Heroon Polytechniou Str., 15773 Zographou, Athens, Greece

tel : +301 7722551, fax: +3017722534

e-mail:ivenieri@cc.ece.ntua.gr

\begin{abstract}
The concept of Intelligent Networks (IN) provides a convenient and future safe way for the rapid introduction of broadband multimedia services. The highly sophisticated design of multimedia services and the increasing demand of system resources creates the need for introducing intelligence on the end-systems. In the IN infrastructure this demand is balanced by the intelligence offered by the network itself. However, the task of deployment of new services or even porting of existing services between IN sub-networks is a tedious task that rarely makes use of the network's intelligence capabilities. The reason is the different implementations of the Service Logic (SL) in IN islands which makes it hard, if not impossible to reuse parts of the software over different IN platforms. This paper proposes ways that enable the remote use of IN capabilities by subscribers which are attached to another IN segment. In this attempt, interconnection issues are resolved using as tools the standard IN modules that is the Broadband Service Control Points (BSCPs) and the Broadband Intelligent Peripherals (B-IPs). Deployment of these modules is based on the use of their service logic execution capabilities and in some cases on an enhancement of their traditional role. To provide the necessary platform and description tools for supporting service logic mobility, service design has been partially based on the concept of movable service logic scripts. Service distribution and service numbering schemes compliant to the network architectures are also provided. Finally, parallel to the description of the proposed models and architectures, practical examples are given.
\end{abstract}

Keywords

Intelligent Networks, Multimedia Networking, Service Logic Mobility, Service Storage Server, IN scripts.

\section{INTRODUCTION}

The original version of this chapter was revised: The copyright line was incorrect. This has been corrected. The Erratum to this chapter is available at DOI: 10.1007/978-0-387-35388-3_42 
The idea of Intelligent Networks (IN) has lead into the development of network tools and infrastructure that allows for fast and efficient introduction of sophisticated services without the need of extended terminal upgrade. However, deployment of new services in new IN locations requires off-line updating of the networks in which services are introduced and porting of these services in a form that is suitable to run over the new network platforms. This is due to lack of a scheme that allows for introduction of services in a generic and platform independent manner over different IN networks.

On the other hand, a serious amount of traffic is imposed on the Intelligent Network in order to transfer information between the service logic execution points that is the Broadband Service Control Point (B-SCP) and the Broadband Intelligent Peripheral (B-IP) during service execution.

The solution to both aforementioned problems seems to be the adoption of a common service creation environment which is platform independent and can facilitate the transport and execution of service logic or service logic parts over different IN modules. This solution introduces the concept of movable service logic modules in the area of multimedia IN service creation and deployment. This concept can be applied to all IN modules directly involved with Service Logic and more specifically to :

1. The B-SCPs in order to exchange executable IN service parts or user related information such as user authentication - authorization information, offering literal service logic mobility over an IN Broadband Network infrastructure.

2. The B-IPs in order to exchange information related to resources used to support service logic mobility offered by the B-SCPs and to reduce the amount of information that needs to be duplicated or updated each time an IN service is introduced or modified.

To fully support the idea of service logic mobility over the network some basic principles need to be adopted. These can be summarized in the following :

- Use of service logic modules based on a platform independent implementation suitable for transport and execution over different IN elements.

- Development of the necessary environment capable of manipulating the transportation of service logic and the migration of service logic execution.

- Development of the necessary security mechanisms which will allow the safe transportation and testing of transported service modules on the host systems and which would guarantee faultless service logic execution.

- Development of a service distribution scheme which would minimize the exchanged information over the network in order to execute an IN service.

The paper is organized as follows. In Section 2 we present the general idea of service logic mobility as it will be used in the rest of this paper together with an example of a moving service. Section 3 gives details about the proposed network and system architectures. In Section 4 the numbering and addressing schemes deployed for supporting the presented architecture are described. Conclusions are summarized in Section 5. 


\section{SERVICE LOGIC MOBILITY IN BROADBAND IN}

The main idea behind the concept of service logic mobility is to provide a scheme that allows the design of services that may be moved between IN modules, even ported to different IN systems. For this reason, the design of these services needs to be based on coherent independent modules which can be combined to produce integrated services. On the functional level this implies the design and implementation of independent re-usable service building modules which can be combined for the design of a service. The description of these modules could be scaled in different levels starting from the Global Functional Plane (ITU Q1203) or the Distributed Functional Plane (ITU Q1214) of the IN architecture. An example of such a service module combination on the Global Functional Level in order to form a service is the integration of an AUTHENTICATION, AUTHORIZATION, FIRST LEVEL SELECTION, PREVIEW, and REGISTRATION, DEREGISTRATION functional modules for providing a Video On Demand Service (Hussmann 1995). As one can see, several of these modules (i.e. AUTHENTICATION, AUTHORIZATION, REGISTRATION, DEREGISTRATION) could be re-used to construct other services. An example could be a Broadband Video Conference service that needs to make use of these modules. Throughout the paper the term 'script' is used in order to identify these modules. In other words a 'script' is an object - oriented self-contained entity that implements integrated and functionally independent parts of an IN service. Scripts can be transferred and executed either in the B-SCP or the B-IP. This is contrary to the narrowband IN approach where the service logic programs reside entirely inside the SCP. Obviously script development should be carried out with platform independent implementation object - oriented tools such as JAVA (Sun 1995) or Safe-TCL (Borenstein 1994).

By making use of the proposed ideas, an infrastructure on which portable services could be provided may be introduced. This infrastructure would allow the porting of a service between IN modules on two levels:

- The first is porting of services between IN modules of the same type such as BSCPs (or even B-IPs). This allows for the movement of services or service building modules (i.e. AUTHORIZATION module) over the IN and also the use of a service or service parts over different IN implementations. Regarding the systems used to facilitate User Interaction such as B-IPs, the same logic would lead into customization of the user interface based on specific user information such as native language, disabled persons options, or even terminal specific information. This way, on one hand there is no need for duplication of services over different IN implementations, while on the other hand the customization of services and re-deployment over different environments based on specific user profiles becomes possible. Movement of services could be achieved by an B-SCP to B-SCP communication while customization of the user interface could be performed by a B-IP to B-IP communication. An example could be the deployment of a Video On Demand 
service made possible over different networks based on the same User Front End and further more using a unique and global user profile meaning customized user - interface based on the native user's language.

- The second is porting of services between IN modules of different type such as B-SCPs and B-IPs. This could help the support of the same services even in environments that are built over different architectures. A possible example could be the deployment of a Video On Demand service which makes use of a B-IP even over an infrastructure that does not deploy a B-IP. This could be realized by reducing the functionality of the offered service related to the use of Special Resources (for example if a video preview option is offered to the user via the B-IP this could be omitted id a B-IP is not present). Another example is the transfer of service logic for the B-IP from central points implemented on B-SCPs.

Of course a coordinator of this service movement is needed in order to supervise the service migration procedure. Special service logic running on the B-SCPs could be used for this purpose. An inherent advantage of this idea of moving services is the easy introduction of new services or even the enhancement of existing ones. The idea of Service Storage Servers (SSSs) based on B-SCPs could be used for this purpose. This implies the implementation of several central B-SCP points, on which available services could be located and from which these services could be transmitted to the endpoint B-SCPs (client B-SCPs) interacting with the users. Therefore, introduction of a new service would rely on uploading of the service logic on the SSSs and notification of the client B-SCPs about the availability of this service. Caching mechanisms for downloading and temporary storage of services on the endpoint B-SCPs could apply reducing traffic based on service transportation requests.

Finally, several mechanisms for handling the requests for locating and downloading of services over the network should apply over the movement of services for ensuring faultless transmission. For this reason, specific service logic parts which could be integrated on the implementation platform of the end systems or embedded in the transmission protocols could be used.

Summing up the presented ideas and before we proceed with methods for supporting such an infrastructure, a simple example of a possible use of a service and the way the proposed infrastructure could handle the request and execution of this service will be presented. This is based on the architecture depicted in Figure 1 . 


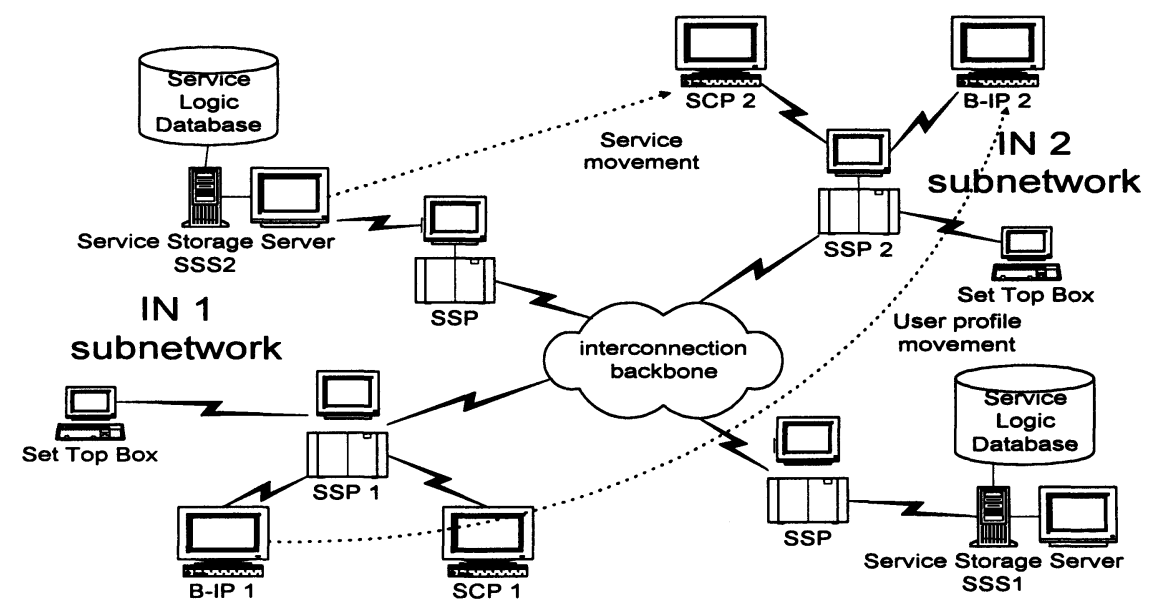

Figure $1:$ An example of a scenario.

Let us assume that a user originated on Intelligent Network 1 is making use of several IN services based on a specific user profile. This profile could determine user settings such as native language, or customized registration Front Ends. This user is moving to IN2, while he wants to make use of a newly introduced service now available in all Service Storage Servers (i.e. SSS2). Let us assume that the user has the related software application installed on his terminal and is now requesting the use of this service. In section 3 we will see how this is possible even without using an IN infrastructure. Once the request for use of this service has been issued, the local Broadband Service Switching Point (B-SSP2) is requesting the execution of this service from the local B-SCP (B-SCP2). The B-SCP is responsible for locating the SSS2 containing the service logic and the user profile for customizing the service provision. The service logic is now downloaded from SSS2 to B-SCP2, while the preferences related to the user profile are transferred from the remote BIP (B-IP1) to the local B-IP (B-IP2). Now the execution of the service is initiated on B-SCP2 while interaction with the user is performed over B-IP2 based on his native language. Furthermore, if we assume that registration to the service is required, this could be performed over the front end specified in the user profile.

\section{THE PROPOSED ARCHITECTURE}

\subsection{Requirements for service logic mobility}

In order to support the idea of movable scripts, we must first provide a suitable architecture on which these scripts will operate. This architecture must comprise of: - An implementation platform on which service logic could be executed independently of the underlying host platforms. The use of JAVA as such a platform seems to be an appropriate solution. 
- A reliable and fast protocol for the transport of service or service modules over the network.

- A Service Storage Database and the related Database Management System for storing the service modules and handling service access requests. This could run on special service storage servers based on B-SCPs which could transmit the services to final destination B-SCPs or B-SSPs upon user request.

- A routing scheme for use during the movement of services. This routing scheme would have a dual use. The first is to provide a way of locating the SSS holding the service parts and the second is to locate the IN modules (BSCPs and B-IPs) holding service user related information. This scheme could be based on the interaction between B-SCPs in order to schedule and route the transport of a service.

- A service logic implementation scheme for providing new services. As already mentioned such a suitable scheme is the use of IN scripts (Patrikakis 1997).

\subsection{System architecture}

Taking into account the study of the proposed architecture, one can see that a distributed architecture for providing a storage and distribution scheme, is the most suitable for supporting such a configuration. In this scheme we can distinguish several IN sub-networks consisting of client modules based on standard B-SCPs and B-IPs. These sub-networks are interconnected through an IN infrastructure serving a dual purpose. First to interconnect the IN sub-networks in order to facilitate the information exchange between these sub-nets and second to interconnect the clients with SSSs located in central points. This infrastructure is depicted in Figure 1and is based on the deployment of the following components :

- The SSS which is built over an IN B-SCP and is used for storing the service logic information. In order to deploy a new IN service or service module, the supporting service logic needs to be uploaded on the server. A special SL daemon running the SSSs is used in order to notify all SSSs about the availability of this service. The server supervisors may decide about the policy of the servers related to the duplication of the service modules locally.

- The client which is also based on an IN module. The functionality of the client is the standard functionality of the host module (B-SCP, B-IP) enhanced with extra functionality in order to connect and download service modules from the SSSs or even from other clients. The client is not aware about the introduction of new services until a request for the use of such a service is issued. At this point, a communication with the closest SSSs takes place and the client is notified about the availability of the requested service.

Following, the architecture on which to base a server and a client system are presented.

\section{A. The Service Storage Server}

The server is located on a central point of the network infrastructure and may serve many IN sub-networks. It is based on the architecture depicted in Figure 2. 


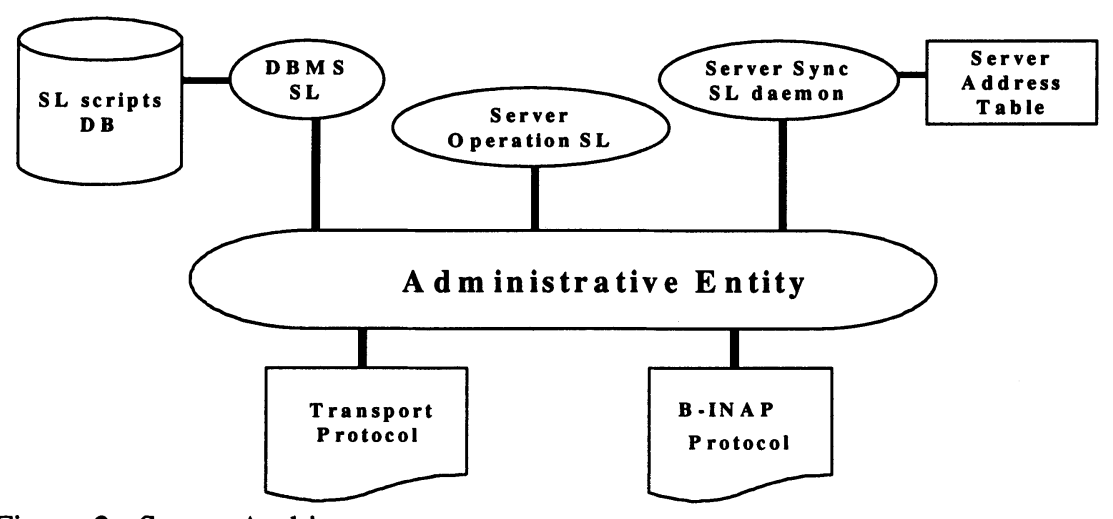

Figure 2 : Server Architecture.

The server consists of a core Administrative Entity which is acting as a manager of the server system. This entity handles all interactions and requests between the different modules of the server. The upper layer interface of this entity is used in order to interact with the service logic instances of the server while the lower interface is used for interfacing the protocols of the server. The service logic instances of the server are the following:

- The Server Operation SL which is the service logic responsible for performing system related operations on the server.

- The Database Management System service logic responsible for managing the database which stores the Service Logic scripts.

- The Server Sync Service Logic Daemon which is a service logic process running continuously on the server and which is used in order to keep the server always aware of the available services. For the latter, a server address table which is updated from the Server Sync SL daemon is used. This table holds the valid addresses of other SSSs on the network together with information about the location of services on these servers.

There are two protocols on the server:

- A transport protocol used for performing uploading and downloading of the service logic scripts from the clients,

- and a Broadband-IN Application Protocol stack (B-INAP) used for interfacing all IN modules (i.e. B-SCPs, B-SSPs).

The service logic scripts on the server comprise of two parts : The first is the main service logic script which runs on the B-SCP and implements the IN service and the other is a service logic script destined to run on the B-IP for supporting the B-SCP operation. The second script instance may be void if no B-IP deployment is necessary. Upon reception of a download request, the service logic scripts are sent to the destination B-SCP and B-IP. The entire procedure is manipulated by the BSCP client which requested the download.

Let us see how the server operates by describing a scenario for the introduction of a new IN service. We will describe the process that takes place on the servers based on the architecture presented in Figure 2. First, the service is uploaded on a Service 
Storage Server and a unique service identification number is assigned to the service. In section 4 we will present the scheme used for identifying services. To do so, the transport protocol is used for uploading the service to the SSS. During the upload of the service, the Database Management System (DBMS) SL is activated for stroring the service logic on the SL scripts database. The service logic is implemented in the form of scripts.

Once the SL for the new service has been uploaded on the server, the Server Sync SL daemon is activated. The daemon sends a message using the B-INAP protocol to all servers listed on the Server Address Table. In case the service logic is not uploaded, the database of the SSS is updated with a link to the server that holds the service logic. Therefore, if a request is sent to an SSS for a service not available at the server's databases, it will be forwarded to the SSS holding this service logic. After this point the SSS is capable of transmitting the service logic to any B-SCP client upon request of this service. As one can see, this architecture enables a distributed storage of services, upon selective duplication of service logic scripts on the SSS databases.

\section{B. The Client}

As it has already been mentioned, the client is a classical IN module based either on an B-SCP or a B-IP. A client may operate also as a server in case a request for downloading a user profile is received. This is necessary to reduce the amount of information maintained by the servers and to distribute it throughout the network on the B-SCPs and B-IP of the various sub-nets. Due to the dual form of the clients, we have two different architectures presented in Figure 3. The first is a reduced architecture based on an B-SCP and is depicted by modules drawn in solid line. The second is based on a B-IP and is depicted by all modules (drawn with solid and dotted lines).

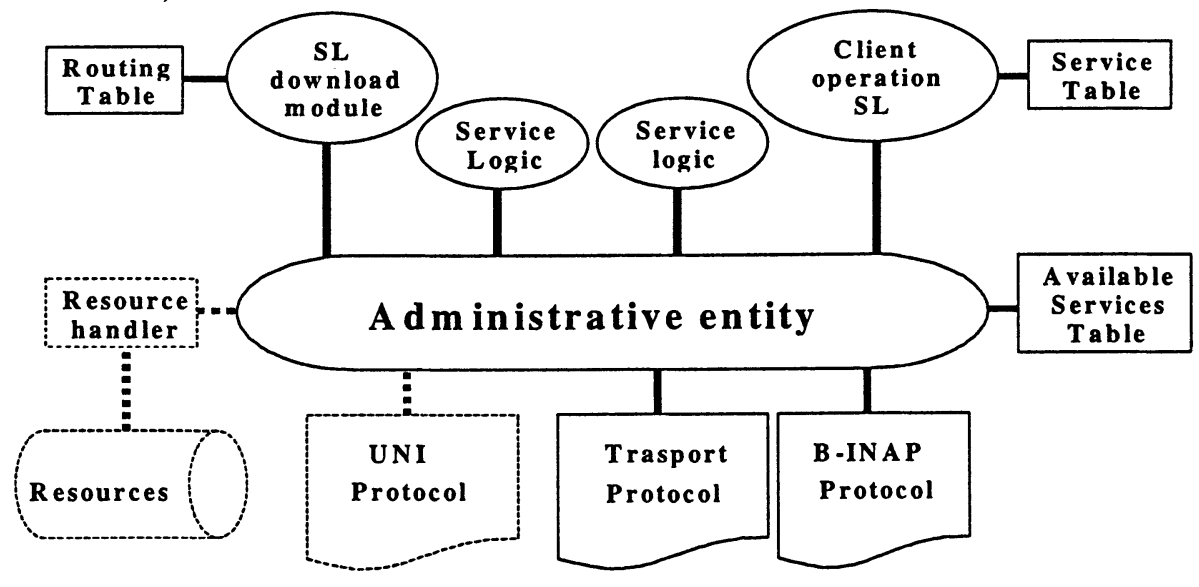

Figure 3 : Client architecture.

\section{B-SCP based client.}


This is the simplest form of client which is based on an B-SCP that may dynamically download and execute a service upon user request. It consists of the following components: A core Administrative Entity which manages all client modules and is responsible for the interaction of these modules. The implementation of the client Administrative Entity is similar to this of the server Administrative Entity. It uses a higher layer interface for the SL instances and a lower level interface for the protocols. The SL instances are the Client Operation SL which is the main SL instance performing system operations on the client and the IN service SLs which are the scripts implementing the services available at the client. The latter may be dynamically updated on the client upon user's request resulting to the availability different service logic scripts on the client at different times. An Available Services Table is kept in order to hold the identity of each supported service on the client. Finally, the SL download module which is used to locate a service on the network if this service is not supported from the client BSCP at the time of request and to manage the download of the service.

The SL download module is also used to access a user profile located on another BSCP client if such a profile is necessary for offering an IN service. Downloading of the service is performed in two steps: First the SL part running on the B-SCP is downloaded, and then if a B-IP SL part exists, the related SL is downloaded on the B-IP connected to the B-SCP by an B-SCP initiated call (optional). A routing table is used to locate the Service Storage Server or another client B-SCP. Regarding the protocols of the client we have a transport protocol used for downloading the service logic scripts, and a B-INAP protocol for interacting with the IN modules (i.e. B-SCPs, B-SSPs).

We will present the operation of an B-SCP client with an example based on the model illustrated in Figure 3. Let us suppose that a user located in a sub-network is making a request for a service. This request is forwarded to the closest client BSCP and an instance of the service is checked to be found on the B-SCP. If the SL for supporting this service is not found on the B-SCP, then the SL download module takes over and the operation of locating the service is initiated. The service location procedure is executed based on the use of the routing table of the client BSCP. The closest (or preferred) SSS address located in this routing table is contacted and a request for this service is sent. If the server contains the SL in its database then the service is downloaded on the B-SCP client. Else the server is transferring the request to another server which has this SL stored on its database. We must remind that if an SL running on a B-IP is necessary for the execution of the service, this is also downloaded to the B-IP corresponding to the B-SCP client. Finally, a check is performed and if the download of a user profile is necessary, the ID of the user that has requested the service is processed from the SL download module to determine the user's home network. The next step is to communicate with the B-SCP in the user's home network and to request the download of the user's profile. Once downloading of the SL for the requested service is finished, the SL instance is set active, the supported services table is updated, and a caching mechanism is initiated for this service. The service logic will remain on the client 
B-SCP within a period in which no request for this service has not been issued. After this period the service logic may be discarded to make room for downloading another service logic script. This way, the service logic instances on the client BSCPs are constantly updated upon user request.

\section{$B$-IP based client.}

This form of client is more complicated than the B-SCP based, and is depicted in Figure 3 by both solid and dashed lines. It uses the same architecture as the B-SCP based client, enhanced with a resource handler for manipulating the B-IP resources, the resources module and a UNI protocol stack (ATM 1994) for supporting the communication with the user. In this form of client, the IN service SL instances are SL programs used to support the service running on the B-SCP and their task is to perform the necessary interaction with the user upon B-SCP request. The SL download module is mainly used for handling any download of SL that is initiated from the related B-SCP once the user has requested a new service. This way, the BIP does not deal directly with SL downloading since this is performed indirectly by the B-SCP. However, there are cases where a specific user profile is required to provide for a customized application front end for an IN service. The client B-IP offers this possibility by using the SL download module in order to contact the remote B-IP client and download this information based on the user profile which is available from the client B-SCP (see previous section). In order to do this a routing table similar to the one used in the B-SCP client is used. This operation implies that each user can be identified uniquely on a global basis as will be presented in section 4 below. The SL instances remain on the B-IP until the related B-SCP asks for withdrawal. The B-SCP caching mechanism is responsible for the definition of the time period for which the SL for a service is present on the B-IP.

Let us present the operation of a B-IP client following the example presented in the previous paragraph for the B-SCP client. We will make the assumption that the service requested by the user involves the use of a B-IP. In this case, after the BSCP SL has been downloaded from the SSS, the B-IP SL is also downloaded and a check is performed to determine whether the service should be provided over a custom user profile or the user wants a customized front end. We us assume that the user asks for a front end based on his native language. Such a front end is available only in his home network based on the home network B-IP resources. For this reason, first the user profile is accessed through the user's home network B-SCP client. Then the B-IP checks on the user profile and contacts the user's home network B-IP in order to download the necessary resources (native language interface). Now the service may be offered over this interface using the local B-IP.

C. User Terminals.

The architecture presented in this paper was designed with the objective to keep service logic mobility transparent to the user's terminal. Only a "Navigation and Download application" is necessary for the terminals, enabling the download of software for deploying new services. This application can be based on widely used front ends that run over non IN networks. In this sense, the software for supporting a new service can be downloaded even from a non IN network (e.g. INTERNET) 
by making use of this navigation and download application before the user has made a request for using a particular service. It is foreseen that special sites will exist where the users can find information on the newly introduced services and download the necessary software. Once the software is installed on their terminals services can be requested from every IN access point globally even through specific user profiles.

\section{ADDRESSING AND NUMBERING SCHEMES}

To support the architecture presented so far, a scheme for addressing the participating IN modules becomes necessary. In this section, possible realisations of such addressing schemes are given.

\subsection{Service Storage Servers addressing}

As mentioned, a Server Address Table is used in each SSS for storing the SSS addresses in the network. This table holds only a subset of all SSS addresses. Whenever a new service is introduced, notification to the SSS relies on the reproduction of notification messages from each server to all servers registered in its Server Address Table. This notification procedure is triggered on the SSS where the service was introduced. From this SSS, the first notification messages to the other SSSs on the network are transmitted. Upon reception of a notification message, each SSS forwards it to all SSS listed on its Server Address Table.

To avoid excessive traffic and useless multiplication of notification messages over the network, each SSS forwards the notification message to the other SSSs only once. Hence, only the original reception of a notification message triggers broadcasting on each SSS, while all other same notification messages received after that are ignored. The notification message encompasses the following information:

- Service Identification Number

- Necessary modules for deployment of the service (B-SCP, B-IP)

- Address of the SSS that holds the service logic for the new service

- A field indicating if the server is the initial server on which the service was uploaded or just a server holding a copy of the service.

Following this service distribution scheme, each SSS that reproduces the service by creating a local copy, inserts its own address on the third field of the notification message (SSS address that holds the service logic). Else the notification message would be reproduced and forwarded as it was received. In Figure 5, an example is presented. The gray filled circle indicates servers that will hold copies of the service while the white filled circle indicates servers that do not hold copies of the service. Finally, the solid line depicts the notifications that will be accepted while the dotted line notifications that will be ignored. 


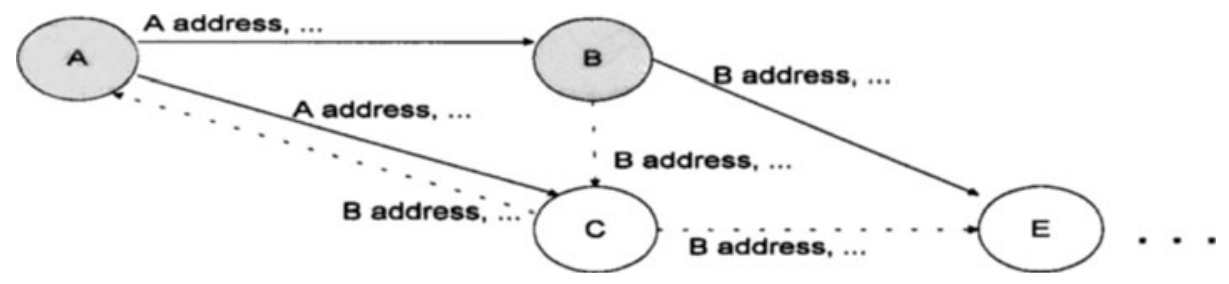

Figure 4 : Example of service notification messages.

Let us suppose that a new service is introduced on SSS A. The Server Address Table of A holds the addresses of B and C. Therefore a notification to these SSSs is issued with the SSS address field set to SSS A address. Server B makes a copy of the service and sends a notification to $\mathrm{C}$ and $\mathrm{E}$ by inserting its address on the server address field of the notification message. Notification to $C$ is ignored since such a notification has already been received from A. SSS C does not create a copy of the service and issues a notification message to $\mathrm{A}$ and $\mathrm{E}$ by keeping the server address field of the notification message which now holds the address of A. Finally SSS E ignores the message of $\mathrm{C}$ since it has already been informed by B. After the exchange of messages for notification of a new service introduction has finished, the following information related to the availability of the new service will exist on the servers address table:

\begin{tabular}{|c|c|c|}
\hline SSS & Type of service copy & Nearest SSS with service copy \\
\hline A & original & A \\
\hline B & copy & B \\
\hline C & none & A \\
\hline E & none & B \\
\hline
\end{tabular}

Table $1:$ Server address table.

In the case of B-SCP clients, a routing table is used in order to access the closest SSS server. Whenever a service request is issued, the closest available SSS is contacted. To overcome failures in service requests in case an SSS in not operating, the addresses of several SSS are available in the routing table together with priority numbers. If a request to an SSS fails, a request to the SSS characterized by the next priority numbered is issued while the SSS which failed to respond is marked as unavailable. The next request starts with the SSS with the highest priority number among the available SSSs while a polling request to the unavailable SSSs is issued. Those who respond are marked as available and are restored to their priority numbers. If an SSS receives a request for a service for which it has no copy, this request is forwarded to the SSS related to this service in the server address table.

This scheme assures the distribution of services and links to servers across the network by referencing a server that is closer to the point where the service is requested. Alternatively to accepting only the first notification message, each SSS could have a certain time period in which all notifications received are accepted and processed for determining the closest SSS. The notification message originating from this SSS is accepted while all others are discarded.. A timer 
initiated after the first notification has been received is used for setting the waiting time for the acceptance of notifications.

\subsection{Client addressing}

Apart from communicating with a server, a client may have to contact other clients in order to request user profile information or resources related to this profile. To do this, the client needs to know the user's home network. This problem is solved by introducing a global user ID number which identifies a user globally and is based on the following scheme :

\begin{tabular}{|l|l|}
\hline Home Network Prefix & User ID \\
\hline
\end{tabular}

On the first part of the address the home network is identified while on the second a user ID is provided. Masking techniques could apply to both fields creating the idea of sub-networks and user groups. As one can see, this technique is similar to the one used in the IP addressing scheme (Socolofsky 1991) which is used for INTERNET accessing. Therefore, whenever a user requests a service, the local BSCP decides about the user home network and treats any requests for user profile download accordingly.

\subsection{Service identification}

Taking into account the distribution scheme presented earlier for the introduction of new services one can see that the service identification scheme has to be dealt from the entity that uploads the service on the first SSS from which distribution and notification about the new service is initiated. Therefore, the service ID for each service or service module is assigned off-line. This is the easiest way in order provide the same service ID request on the software necessary to be deployed on the terminals.

\section{CONCLUSIONS}

This paper has investigated key architectural and design issues regarding network infrastructure related to the supporting of IN services over different IN implementations. The architectural schemes proposed in this paper provide the basis for an IN design that allows for quick and easy introduction of new IN services. The idea behind these architectures is the use of IN modules described in current IN recommendations, enhanced with service logic mobility support features. Service numbering and service distribution schemes for supporting service introduction under the presented architecture were also provided. Finally, implementation tools, as well as transport and signaling protocols capable of satisfying requirements posed by the proposed architecture were also proposed. The authors of this paper are currently engaged in the development of B-SCPs and B-IPs and have based the presented ideas on the experience gained in the context of ACTS INSIGNIA project.

\section{ACKNOWLEDGEMENT}


This work was performed under the context of the EU ACTS Project MARINE (AC-340). The opinions appearing in this paper are those of the authors and not necessarily of the other members of the project consortium.

\section{REFERENCES}

ATM User-Network Interface Specification V3.1 (1994) "af-uni-0010.002"

Borenstein N. S. (1994) "E-mail with a mind of its own: The Safe-Tcl Language for Enabled Mail", ULPAA, Barcelona

Hussmann H., Straten G.v.d., Theimer Th., Totzke J. (1995), "An IN-based Implementation of Interactive Video Service," in Proceedings of ICC'95, Seattle

ITU Recommendations Q1203, "Intelligent Network Global Functional Plane architecture"

ITU Recommendations Q1214, "Distributed Functional Plane for Intelligent Network Capability Set 1"

Patrikakis Ch. Z., Venieris I.S., Protonotarios E. N., (1997) A modular Architecture for Broadband Multimedia Services Intelligent Peripherals", IS\&N 97,15, Como

Socolofsky T., Kale C., (1991) A TCP/IP Tutorial, RFC1180

Sun Microsystems (1995) The Java Language Environment: A White Paper

\section{BIOGRAFY}

Charalampos Z. Patrikakis was born in Athens, Greece, in 1970. He received the Dipl.-Ing. degree from the Electrical Engineering and Computer Science Department of the National Technical University of Athens (NTUA), Greece in 1993 and the Ph.D. degree from the National Technical University of Athens in 1997. Mr. Patrikakis is a research assistant in the Telecommunications Laboratory of NTUA performing research in the area of B-ISDN access networks, signaling, multimedia service design, and Intelligent Network technology. He has participated in several European Union projects and is currently involved in the INSIGNIA AC068 project. He is a member of IEEE and the Technical Chamber of Greece.

Spyros E. Polykalas was born in Kefalonia, Greece, in 1971. He received the Dipl. -Ing. Degree from the Electrical Engineering Department of the University of Patras, Greece in 1994. Since 1994 he is a Ph.D. candidate in the Electrical and Computer Engineering Department of the National Technical University of Athens (NTUA), Athens, Greece and research associate of the Telecommunications Laboratory. His research interests are in the area of signalling, Intelligent Networks, Personal Communications, performance evaluation and modeling. $\mathrm{He}$ has participated in several European Union ACTS projects and is currently involved in INSIGNIA AC068 and MARINE AC340 projects. He has received 
several national awards for the entire five years of his undergraduate study. Mr. Polykalas is a member of IEEE and the Technical Chamber of Greece.

Iakovos S. Venieris received the Dipl. -Ing. degree from the University of Patras, Patras, Greece in 1988, and the Ph.D. degree from the National Technical University of Athens (NTUA), Athens, Greece, in 1990, all in electrical and computer engineering. He is currently an Assistant Professor in the Electrical and Computer Engineering Department of NTUA. His research interests are in the fields of B-ISDN, telecommunications software, internetworking, signalling, network management, modelling, performance evaluation and queueing theory. He has been exposed to standardisation body work and has participated in several European Union and national projects. Dr. Venieris is a member of IEEE and the Technical Chamber of Greece. 